\title{
Correction
}

\section{On the Injectivity of the Map of the Witt Group of a Scheme into the Witt Group of its Function Field}

\author{
F. Fernández-Carmena
}

Arturo Soria 310 (3A), E-28033 Madrid, Spain

Math. Ann. 277, 453-468 (1987)

In the published version of the article a whole portion of the text is missing starting on p. 465, line 6. This portion of the text includes the statement of a Proposition, together with the beginning of its proof; it is the rest of this proof which occupied the last pages (465 through 468) of the article. What follows is the missing text of the paper, from the beginning of Remark 18 to the line preceding Diagram (7) on p. 465. There are no changes from that point on.

Remark 18. As suggested by Proposition 14, the construction of [9, Sect. 7] can be carried out for split formations, thus getting a map $D: S p W_{1}^{1}\left(\mathbb{M}_{1}\right) \rightarrow W(X)$; from the way this map is defined, it follows that, for any split formation $u=\left(\mathscr{F} \oplus \mathscr{F}^{\wedge}, \phi\right.$; $\mathscr{F}, \mathscr{G})\left(\phi\right.$ : the hyperbolic form), any extension of $\mathscr{F}^{\wedge}$ (or $\left.\mathscr{F}\right)$ by locally free sheaves defines a split resolution of $u$ [i.e., a resolution which satisfies the splitting property (3) of Definition 15 globally], and $D$ sends $u$ to the class in $W(X)$ of the bilinear space associated to that resolution. This class in $W(X)$ naturally does not depend on the choice of the extension of $\mathscr{F}^{\wedge}$. Our notion of resolution is analogous to a map in $K$-theory which goes from $K_{1}$ to $K_{0}$ [see the proof of (3.1) above], and in the affine case, it has a more intrinsic interpretation in the context of Poincaré complexes (see [12]).

Proposition 19. Let $u=(\mathscr{M}, \phi ; \mathscr{F}, \mathscr{G})$ be a resolvable formation, and fix a resolution of $u$ with associated bilinear space $\mathcal{N}$. Let $v$ be the split stabilization of $u$ constructed in Proposition 12. Then $v$ has a split resolution whose associated bilinear space determines the same element in $W(X)$ as $\mathcal{N}$, and, with the notation of Remark 18, $D([v])=[\mathcal{N}]$.

Proof. Let the resolution of $u$ be

$$
\begin{aligned}
& \mathscr{R} \mapsto \mathscr{L} \rightarrow \mathscr{Q} \\
& \mathfrak{I} \mathfrak{I}^{n} \quad \mathfrak{I} \\
& \overline{\mathscr{Q}} \stackrel{\mu}{\rightarrow} \overline{\mathscr{L}} \underset{\boldsymbol{\imath}}{\rightarrow} \overline{\mathscr{R}} \\
& \downarrow \downarrow \text { tw } \\
& \mathscr{F} \mapsto \mathscr{M} \rightarrow \mathscr{F}^{\wedge}
\end{aligned}
$$


and let $\phi_{\bar{L}}$ be the form on $\overline{\mathscr{L}}$; then $h$ is the adjoint of the form defined on $\mathscr{L}$ by restricting $\phi_{\bar{L}}$. Consider the following diagram:

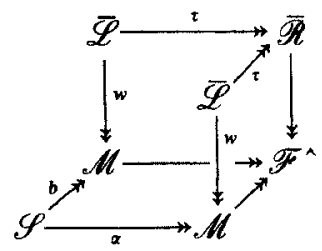

[from diagram (1) above; notice that, in our case, $\mathscr{K}^{\wedge}$ of (1) becomes $\mathscr{M}$, as indicated in the proof of Proposition 12]. Call $\mathscr{S}_{2}$ the pullback

$$
\begin{array}{ccc}
\overline{\mathscr{L}} & \rightarrow & \overline{\mathscr{R}} \\
\uparrow & \uparrow \\
\mathscr{T}_{2} & \rightarrow & \overline{\mathscr{L}}
\end{array}
$$

Then $\mathscr{S}_{2}$ surjects onto $\mathscr{S}$, and we have a commutative cube:

[The text now continues with diagram (7).] 